\title{
Hacia un Modelo de Metagobernanza para GipuzKoa: el CASo de Etorkizuna ERAIKIZ
}

TOWARDS A METAGOVERNANCE MODEL FOR GIPUZKOA: THE CASE OF ETORKIZUNA ERAIKIZ

\author{
Xabier Barandiaran \\ Departamento de Trabajo Social y Sociología, Universidad de Deusto, Donostia-San Sebastián \\ xabier.barandiaran@deusto.es
}

\begin{abstract}
Resumen: El presente artículo está orientado al análisis y descripción del programa de gobierno Etorkizuna Eraikiz implementado por la Diputación Foral de Gipzukoa. Este artículo aborda este análisis desde un ejercicio reflexivo centrado en los conceptos de gobernanza y meta-gobernanza como manifestaciones de las nuevas condiciones para el ejercicio del buen gobierno y la competitividad territoriales. Etorkizuna Eraikiz se erige como el primer paso para el establecimiento organizado de las condiciones para una nueva gobernanza del territorio más colaborativa y abierta. El objetivo de este programa es visualizar y garantizar los mecanismos necesarios para la institucionalización de un nuevo sistema de deliberación compartida con la ciudadanía y los principales agentes e instituciones territoriales de Gipuzkoa para afrontar sus principales retos de futuro.
\end{abstract}

Palabras clave: meta-gobernanza, gobernanza, innovación pública, Gipuzkoa, políticas públicas, competitividad territorial.

\begin{abstract}
The present article is focused on the analysis and description of the government program Etorkizuna Eraikiz implemented by the Provincial Council of Gipzukoa in the Basque Country. This article approaches this analysis from a reflective exercise focused on the concepts of governance and meta-governance as manifestations of the new conditions for the exercise of good governance and territorial competitiveness. Etorkizuna Eraikiz stands as the first step for the organized establishment of the conditions for a new governance for a more collaborative and open territory. The objective of this program is to visualize and guarantee the necessary mechanisms for the institutionalization of a new system of shared deliberation with the citizens and the main agents and territorial institutions of Gipuzkoa to face their future socioeconomic challenges.
\end{abstract}

Palabras clave: meta-governance, governance, public innovation, gipuzkoa, public policies, territorial competitiveness.

\section{Introducción}

Etorkizuna Eraikiz -construyendo el futuro- es un programa político enfocado hacia la creación de una estrategia de meta-gobernanza (Jessop, 2003; Kooiman, 2003; Sorensen, 2006; Meuleman, 2008; Christopoulos et al., 2012) y competitividad territoriales (Camagni, 2002; Moulaert \& Sekia, 2003; Martin et al., 2008; Valdaliso \& Wilson, 2015) implementado por la Diputación Foral de Gipuzkoa entre 2015-2019. Esta estrategia se compone de una serie de acciones para abordar los futuros retos socioeconómicos y políticos del territorio Gizpuzkoano. Esta iniciativa se orienta hacia la construcción de un nuevo escenario de políticas públicas capaces de responder coyuntural, endógena y organizadamente a los déficits y desafíos democráticos de nuestro tiempo a través de la ampliación de los escenarios de deliberación política, yendo "mas allá" de los sistemas de Gobernanza más tradicionales (Jessop, 2003; Torfing et al., 2012).

Etorkizuna Eraikiz se concibe también como una compleja apuesta integrada por distintas iniciativas de gobernanza abierta y colaborativa (Lathrop \& Luma, 2010; Sorensen \& Torfing, 2007; Emerson et al., 2011) consistente en un ejercicio de experimentación activa a través de 
proyectos de interés general y una prospectiva compartida con los agentes público-privados del territorio Gipuzkoano para la construcción de una nueva agenda política. Esta propuesta pretende encarar los retos socioeconómicos de Gipuzkoa para reorientar su futuro económico, político, social y cultural desde un ejercicio reflexivo que permita el fortalecimiento organizado de la sociedad Gipuzkoana en el medio y largo plazo.

Asimismo, esta estrategia se inscribe en un contexto donde el incremento de la complejidad de los sistemas sociales (Lipset, 1959; Luhmann, 1982) y los programas políticos derivados de la creciente interdependencia de los distintos niveles de gobierno, la diversidad de actores y agentes políticos involucrados y el aumento de las áreas de actuación política (Jessop, 2004; Papadopulos, 2007; Duit \& Galaz, 2008), nos obliga a reflexionar sobre la importancia de implementar iniciativas políticas capaces de aglutinar en un solo programa político conceptos cómo los del "buen gobierno", la "gobernanza abierta", la "gobernanza interactiva" o la "gobernanza colaborativa" (Agranoff \& McGuire, 2003; Borras \& Jacobsson, 2004; O’Flynn \& Wanna, 2008; Meuleman, 2008; Lathrop \& Luma, 2010; Emerson et al., 2011) .

Como consecuencia de estos cambios, el término meta-gobernanza (Jessop, 2003; Kooiman, 2003; Sorensen, 2006; Meuleman, 2008; Christopoulos et al., 2012) o "gobernanza de la gobernanza" sirve al análisis de todas aquellas actividades políticas enmarcadas en las nuevas prácticas de gobierno no solamente vinculadas al New Public Management (Ferlie et al., 1996; Bovaird \& Loffler, 2009; McLaughlin et al., 2002), sino también a contextualizar y cuestionar los nuevos procesos de gobernanza publico-privada o de gobernanza en red (Jones et al., 1997; Sorensen \& Torfing, 2007; Provan et al., 2008) adoptados hoy en día por muchos gobiernos (Meuleman, 2008; Torfing et al., 2012). Es decir, ofrece la oportunidad de reorganizar los nuevos criterios y las condiciones para el buen gobierno del territorio Gipuzkoano (Jessop, 2003) desde la creación de nuevos espacios de reflexión $\mathrm{y}$ deliberación compartida sobre sus necesidades futuras. En este sentido, la importancia del concepto de "meta-gobernanza" radica en su aproximación más holística y ordenada para definir y encarar todas las iniciativas, desafíos, programas y procesos de gobernanza futuros de Gipuzkoa.
Este salto aparentemente conceptual que produce la idea de Gobernanza en realidad nos brinda la oportunidad para hablar de aproximaciones y diseños políticos e institucionales que, dentro de los nuevos modelos de gobernanza colaborativa (Agranoff \& McGuire, 2003; O'Flynn \&Wanna, 2008), permiten ampliar el espectro de ideas y prácticas más previsoras y conscientes de los límites del sector público, y los desafíos y condicionamientos políticos, institucionales, sociales y económicos del contexto global que padecemos y vivimos en la actualidad (World Bank, 2000).

El presente artículo se centra en comprender cómo el rol cambiante del liderazgo público en las distintas configuraciones institucionales y campos políticos del contexto actual han dado pie a la transformación de las condiciones estructurales y los mecanismos de gestión pública y política de la Diputación Foral de Gipuzkoa. El objeto de esta reflexión es tratar de interpretar estos cambios aplicándolos a la descripción y el análisis del programa Etorkizuna Eraikiz.

Este artículo se divide en tres secciones: en primer lugar, abordaremos los conceptos de Gobernanza y Metagobernanza desde los cambios sociopolíticos sufridos como resultado de la crisis de legitimidad y la pérdida de confianza (Bouckaert, 2012a, 2012b) en las administraciones públicas; en segundo lugar, abordaremos, la importancia otorgada a los mecanismos de competitividad regional y territorial presentes en este proceso; y en tercer y último lugar profundizaremos en el diseño, la aproximación metodológica y los contenidos de Etorkizuna Eraikiz.

\section{Reflexiones sobre la Gobernanza y la Metagobernanza}

Los procesos de planificación estratégica de la Diputación Foral de Gipuzkoa y el diseño del programa Etorkizuna Eraikiz se inscriben tanto en lo que podemos concebir como las perspectivas sobre la Gobernanza Territorial (Bukowski et al., 2003; Lidström, 2007; Faludi, 2012) como en las lógicas institucionales que ahondan en la búsqueda de procesos de planificación más trascendentales. Por esta razón, conviene hacer un breve acercamiento para comprender y distinguir de qué hablamos cuando utilizamos los conceptos de Gobernanza y Metagobernanza (Jessop 1998, 
2002; Rhodes, 1997,1998 2007; Kjaer, 2004; Bovaird \& Löffler, 2009; Beuir, 2012).

Desde hace ya algunas décadas, la Gobernanza se ha erigido como un concepto clave tanto en la agenda política como en el contrato social europeo. Los criterios de eficacia, eficiencia, transparencia y responsabilidad tan añorados hoy por las buenas prácticas de gobierno, se han visto reñidos con el incremento de la complejidad sistémica y relacional, y con la pérdida de legitimidad de la gestión política, resultando en el conflicto y en el deterioro de las relaciones existentes entre la ciudanía y sus gobiernos; una paradoja que frente a la retórica de las herramientas promovidas por el concepto de Gobernanza y sus buenas intenciones, entrañan numerosas dificultades a la hora de ser puestas en práctica (Atkinson \& Coleman, 1992; Rhodes, 1997; Fukuyama, 2013) en sus diferentes niveles gubernamentales -Europeo, regional, local-, trascendiendo así los espacios y los escenarios tradicionales de representación política.

Además, el concepto Gobernanza se inspira en la necesidad de un cambio progresivo de actitudes que, más liberadas de las rígidas dicotomías de los gobiernos tradicionales, implique las comparticiones gobernativas de las distintas demarcaciones, de modo que diluyan sus antiguas limitaciones entre las responsabilidades administrativas exigidas por los nuevos espacios de la vida social, económica y política de nuestra actualidad (Rhodes, 1997; Stoker, 1995). Con razón escribe Castells que "los sistemas políticos están sumidos en una crisis estructural de legitimidad, hundidos de forma periódica por escándalos, dependientes esencialmente del respaldo de los medios de comunicación y del liderazgo personalizado, y cada vez más alejado de la ciudadanía" (Castells, 1997: 33).

Los cambios en los escenarios políticos, económicos e institucionales (crisis energética, crisis económica, crisis productiva, crisis política) ocurridos en Europa y Estados Unidos durante la década de los años 70 y 80 del siglo pasado con los gobiernos neoliberales ${ }^{l}$ de Ronald Reagan y Margaret Thatcher, aceleraron el incremento de la complejidad político-institucional provocando, en el proceso, una crisis de las herramientas políticas

\footnotetext{
${ }^{1}$ La referencia al neoliberalismo no pretende atribuir en ningún caso un sesgo ideológico al desarrollo de este artículo sino que pretende describir un periodo histórico clave en la historia del pensamiento político de finales del siglo XX.
}

tradicionales, dificultando y diversificando a gran escala los procesos de toma de decisiones, el diseño de políticas públicas $\mathrm{y}$, por tanto, causando el debilitamiento de los mecanismos que posibilitaron el anclaje del Estado de Bienestar Keynesiano tras la Segunda Guerra Mundial (Harvey, 2007).

Este replanteamiento introdujo una serie de obstáculos agravando las disfunciones políticas de los sistemas tradicionales de gobierno. De acuerdo con Crozier et al., (1975) este proceso ha sido producto, precisamente, del éxito del sistema democrático.

Desde este contexto, el concepto de Gobernanza ha ido derivando en ámbitos de actuación específicos demandados por (Luna, 2015):

- $\mathrm{Su}$ capacidad para involucrar a actores y agentes diversos, ya sea desde perspectivas transversales, (públicas, privadas, o de la sociedad civil) y/o a distintos niveles dinámicos de colectivos internacionales, nacionales, regionales, provinciales $\mathrm{o}$ municipales.

- Por la necesidad de articular la complejidad de esta variedad inter-institucional y multidisciplinaria desde agrupaciones conectadas y organizadas en red.

- O por la voluntad de incluir a la sociedad civil en los procesos de toma de decisiones y en la asunción y asimilación de responsabilidades relacionadas con la eficacia y flexibilidad que dichos procesos conllevan.

- Asimismo, por la promoción de una "ética pública", basada en valores y comportamientos, como la transparencia y el rigor, a cargo de todos los actores, agentes e instituciones involucrados.

- Y, finalmente, por la autonomía requerida tanto como capacidad para la toma de decisiones como para la utilización de herramientas alternativas a los hábitos burocráticos y administrativos más clásicos.

La nueva combinación exitosa de estos ámbitos ha dado lugar a un incremento de las relaciones de interdependencia organizacional, a la multiplicación de las redes de intercambio de recursos e ideas, y a la intensificación de sus procesos de negociación. Asimismo, ha aumentado el grado de autonomía de las 
estructuras intermedias de gobierno con respecto al Estado, difuminando también el poder de éste último tanto desde el punto de vista macro, a través del nuevo poder otorgado a los mercados y las estructuras supranacionales, como desde las microestructuras de gobierno regionales y municipales, fragmentando y diversificando cada vez más el sistema relacional.

En este sentido, de acuerdo con Rhodes "la gobernanza se refiere al gobierno a través y entre redes" (Rhodes, 2007:1246) lo cual atribuye al concepto de Gobernanza un carácter colaborativo, interactivo y abierto. La complejidad de esta relación de factores nos obliga a pensar en procesos y perspectivas que sean capaces de ir más allá de las consecuencias y tendencias democráticas antes descritas, desde la importancia otorgada a la generación de redes entre el Estado, el mercado y la sociedad civil.

De este modo, la implementación de las políticas públicas dependen del gobierno que esté en el poder y de la capacidad del mismo para mantener las redes creadas por otros gobiernos o para construir nuevas redes de intercambio (Jessop, 1998, 2002). Está dinámica nos ha conducido a pensar en la importancia de los procesos de innovación en el sector público (Windrum \& Koch, 2008; Bason, 2010; Barlett \& Dibben, 2010) y en el diseño de estas políticas. De acuerdo con Jessop (2002), dado el intercambio de la complejidad estructural y la opacidad del mundo social, el fracaso es el resultado más factible de los distintos niveles de gobierno por alcanzar múltiples objetivos durante un horizonte temporal y espacial extendido, obviando el tipo de mecanismo o modo de coordinación adoptado (Jessop, 2002:5).

La incapacidad y las dificultades de tener éxito a través de la aplicación completa de estos programas coordinados de actuación política, termina por centrar los discursos en la retórica de la coordinación, la gobernanza y los partenariados, en vez de reconocer que la aplicación de estos programas y políticas están, en realidad, ayudando a construir una nueva estructura institucional $o$ una nueva forma de hacer política a largo plazo que está por encima del éxito o del fracaso de actuaciones concretas (Jessop, 2002:5).

\section{Hacia una nueva metagobernanza del territorio}

Este hecho nos conduce a la necesidad de crear meta-estructuras organizacionales de coordinación (Alexander, 1995; Jessop, 1998, 2002); es decir, estrategias de meta-gobernanza capaces de "organizar las condiciones para la gobernanza" (Jessop, 2002:5). Ello implicaría reflexionar sobre la gobernanza y sus mecanismos desde un sentido más amplio, considerando la re-articulación dirigida de sus distintas manifestaciones a través de la creación de estructuras y políticas que van más allá de los modos usuales, velando por la supervisión y coordinación del conjunto.

De acuerdo con Jessop, "la metagobernanza es la gestión de la complejidad, desde la creación de las condiciones estructurales que posibiliten la conjugación del mercado, la ruptura o la inclusión de las jerarquías del poder y las redes" (Jessop, 2002:5). En definitiva, las estructuras de meta-gobernanza implican la capacidad para crear estructuras de gobierno que tengan en consideración los tres sistemas, es decir, la cosmovisión coordinada, eficiente y efectiva del "mercado", las "jerarquías" y las "redes" desde su interconexión, en muchas ocasiones conflictiva (Jessop, 1998; Meuleman, 2008).

Por adoptar una definición del concepto de meta-gobernanza que sea capaz de integrar todas las dimensiones mencionadas, nos quedaremos con la aportación de Louis Meuleman (2008), que entiende la meta-gobernanza como "los medios a través de los cuales producimos algún grado de gobernanza coordinada, desde el diseño y la gestión combinada de gobernanza jerárquica (top-down), de mercado y en red, para obtener los mejores resultados posibles desde el punto de vista de aquellos que son responsables del rendimiento de las organizaciones del sector público: los gestores públicos como metagobernantes" (Meuleman, 2008: 68).

De acuerdo con la reflexión adoptada Christopoulos et al, (2012), en la definición de Meuleman, la meta-gobernanza adquiere un mayor potencial en la gestión de las circunstancias contextuales y específicas, seleccionado las prácticas que mejor se adaptan a los factores institucionales, sociales, políticos y culturales, de los distintos países, regiones y comunidades (Christopoulos et al., 2012: 32). Postura que también es reconocida por Engberg y Larsen (2010) pero desde su énfasis en aquellas 
aportaciones provenientes de la sociedad civil (bottom-up), capaces de reconducir las inconsistencias de determinadas prácticas gubernamentales implantadas verticalmente (Christopoulos et al., 2012; Engberg \& Larsen, 2010).

Finalmente, es importante reconocer también dentro de estos procesos meta-gubernamentales, los mecanismos de gobernanza quasi-mercantil dictados por el New Public Managment (Ferlie et al., 1996; McLaughlin et al., 2002), cuyo mayor exponente es el ahorro y la gestión eficiente y eficaz del gasto público; los acuerdos de colaboración público privada establecidos especialmente a través de procesos de planificación estratégica; y la estructuración de nuevas redes inter e intra-organizacionales entre distintos actores.

Todas las tipologías mencionadas pueden ser reconocidas en el programa Etorkizuna Eraikiz cuya mayor aspiración es, precisamente, la institucionalización de un modelo de gobernanza colaborativo que no sólo defina una agenda pública común de ámbitos de actuación relacionados con la competitividad, la cohesión y la sostenibilidad del territorio Gipuzkoano, sino también con el fortalecimiento de las capacidades contextuales del propio territorio para decidir y enfrentar sus propios retos de futuro.

\section{La importancia de la Competitividad Territorial}

En este contexto, vislumbramos también en todo proceso de gobernanza, la dispersión, polarización y fragmentación de los poderes gubernamentales que se traduce en la nueva capacidad de los territorios y sus gobiernos regionales para competir y adaptarse a los retos globales desde estrategias y políticas locales (Robertson, 1995). Este componente a la vez meta-gubernamental y competitivo es orientado también hacia la concepción del territorio como agente fundamental de actuación en el propio proceso de desarrollo, desde la potenciación de la competitividad y la innovación territoriales (Camagni, 2002; Moulaert \& Sekia, 2003; Crevoiser, 2004; Martin et al., 2008; Valdaliso \& Wilson, 2015). Los sistemas regionales de innovación (Moulaert \& Sekia 2003), por ejemplo, han constituido un gran pilar en la mayoría de procesos y modelos de gobernanza territorial descritos. Asimismo, han dado lugar a procesos de descentralización política y administrativa en el marco de los viejos Estados Nacionales.

En el caso de Euskadi, su marco institucional ha posibilitado el establecimiento de una nueva cultura de gobernanza local en la cual las redes asociativas y de colaboración han instaurado una nueva "lógica de intervención pública" capaz de estructurar un nuevo modelo para la integración de la sociedad civil y la cooperación público-privada (Estensoro \& Zurbano, 2010:133).

Tanto el modelo regional como las corrientes regionalistas beben de los nuevos modelos de gobernanza para potenciar tanto la autonomía como la competitividad de sus demarcaciones territoriales. (Dickinson, 1964; Keating, 1998, 2002). La región al igual que el territorio, no puede desprenderse, de su carga política, ya que como sistema de acción (Luhmann, 1995) tiene ciertas implicaciones en la distribución del poder y en el contenido de las políticas públicas que lleva a cabo. Aspectos tales como su limitaciones territoriales, el sistema institucional que la caracteriza, ya sea como gobierno regional o como conjunto institucional, y su rol como agencia económica, política o social, a nivel nacional o internacional, son factores que deben tenerse en cuenta a la hora de estudiar la conformación socio-histórica que la define y construye (Keating, 1997: 383).

Según Sorensen \& Torfing (2012), estos procesos unidos al cada vez más acusado énfasis en los procesos de innovación del sector público (Windrum \& Koch, 2008; Bason, 2010; Barlett \& Dibben, 2010) han sido influenciados por tres importantes factores históricos: en primer lugar, por la creciente presión, demanda y expectativas ciudadanas dirigidas hacia el sector público en forma de soluciones y nuevos servicios y el acceso limitado a los recursos, agravado por la crisis económica global en el 2008.

En segundo lugar, por la acentuación de una serie de problemas de orden mundial como el cambio climático, la reducción de la pobreza, la seguridad, los procesos de planificación urbana y la necesaria potenciación de los ámbitos rurales. Problemáticas, todas ellas, de gran calado que han intensificado la complejidad de los procesos y herramientas necesarias para solventarlos y que requieren de la reducción de los niveles de riesgo y los conflictos entre todos los agentes implicados.

$\mathrm{Y}$ en tercer y último lugar, el propio proceso de globalización ha derivado asimismo en la nueva construcción de los discursos basados en 
la competitividad territorial (Camagni, 2002; Moulaert \& Sekia, 2003; Martin et al., 2008; Valdaliso \& Wilson, 2015) del cual participan gobiernos, regiones y localidades de todo el mundo por mejorar sus capacidades de innovación, como único vehículo capaz de lidiar con los rápidos procesos de cambio y la acelerada y necesaria adaptación a los mismos (Sorensen \& Torfing, 2012: 3).

Estos factores han sido determinantes en las nuevas perspectivas surgidas en torno al concepto de innovación colaborativa (Torfing, 2016) y a la Nueva Gobernanza Pública (New Public Governance) (Osborne, 2008, 2010) dando como resultado la dinamizadora creación de libros blancos sobre la innovación, laboratorios de innovación pública y social, $\mathrm{y}$ un mayor protagonismo y responsabilidad otorgados a los gestores y empleados del sector público, causando además la creación de nuevos programas de formación centrados en el "aprendizaje a lo largo del ciclo vital" (Life-long learning) ( Field, 2000; Sorensen \& Torfing, 2012: 3).

Uno de los mayores exponentes de este mestizaje entre las nuevas estructuras metagubernamentales y los procesos de competitividad ha sido la creación del Mindlab ${ }^{2}$ en Dinamarca o el concepto de Big Society promovido por el Gobierno de David Cameron en Gran Bretaña (Penin et al, 2015; Foxwell, 2015), centrado en la visión de la ciudadanía y la sociedad civil como un recurso y un socio clave en el proceso de innovación del sector público. Podemos encontrar todos estos elementos en el programa Etorkizuna Eraikiz sobre el que discutiremos en los siguientes apartados.

\section{El diseño de Etorkizuna Eraikiz}

Etorkizuna Eraikiz es producto de un proceso de reflexión y planificación estratégica elaborado por la Diputación Foral de Gipuzkoa para la reorientación de las políticas públicas y la innovación del Sistema de Gobernanza Institucional Gipuzkoano, fortaleciéndolo, dinamizándolo y adaptándolo a los nuevos retos

\footnotetext{
${ }^{2}$ Mindlab es una unidad de innovación pública interministerial creada por el gobierno Danés para promover la innovación colaborativa entre los diferentes agentes y organismos ministeriales y las empresas para responder a nuevos desafíos sociales. Otros ejemplos parecidos pueden ser encontrados en la 27e Región en Francia o Kennisland en Holanda.
}

demográficos, económicos, sociales, productivos y medioambientales, desde la defensa del bienestar y la sostenibilidad territoriales.

Esta estrategia también responde al reto de la Diputación Foral de Gipuzkoa para aportar soluciones de forma colaborativa, sumando conocimientos y experiencias que ayuden a construir conjuntamente un futuro compartido para Gipuzkoa. Este proceso de deliberación ha contado con la participación de las organizaciones, las empresas, las asociaciones, las universidades y la sociedad civil del territorio Gipuzkoano

Este programa se concreta en el diseño de una estrategia participativa a través de la reflexión entre distintos agentes y expertos, valorando los procesos de aprendizaje territorial y la incorporación a redes regionales y polos de innovación más competitivos y avanzados. En este sentido, podemos concebir este programa como el primer paso para el establecimiento organizado de las condiciones para una nueva gobernanza, es decir, como un programa meta-gubernamental capaz de visualizar y garantizar los mecanismos necesarios para un sistema de gobernanza institucional nuevo y consolidado en el largo plazo.

Esta dinámica de planificación surge en el marco del Plan Estratégico de Gestión 2015-2019 de la Diputación Foral de Gipuzkoa en el cual se establecen las bases para el desarrollo de una serie de proyectos, entre los cuales destacan para nuestro análisis, una Estrategia de Buena Gobernanza y un Programa de Cultura Política y Profundización Democrática, concretados en los siguientes objetivos específicos (Diputación Foral de Gipuzkoa, 2016:20):

- La transformación de las formas de gobernar y hacer de la Buena Gobernanza la seña de identidad de la Diputación Foral de Gipuzkoa.

- Profundizar en las actitudes, valores y comportamientos democráticos de la ciudadanía, de los representantes institucionales y de la sociedad organizada.

Estos proyectos quedan reflejados en los objetivos generales más importantes del Plan, entre los que conviene resaltar la Reinvención del Gobierno de Gipuzkoa "como motor que impulse el desarrollo y la transformación del territorio, estableciendo un compromiso corporativo y transversal con el cambio cultural y organizativo que alcance y exija 
a cada persona y área de la Diputación Foral de Gipuzkoa; abriendo cauces ágiles de participación ciudadana, colaboración multiagente y transparencia” (...) “...extendiendo $y$ homogeneizando nuevas prácticas avanzadas de planificación, gestión y evaluación aplicables a cada una de sus políticas públicas..." (Diputación Foral de Gipuzkoa, 2016:15).

Dentro de este marco de actuación y reflexión surge el programa Etorkizuna Eraikiz enfocado a enfrentar los retos de futuro del territorio desde dos ideas básicas: por un lado, la modernización de Gipuzkoa a través de nuevos proyectos económicos, sociales y culturales; y por otro lado, el diseño de los ámbitos estratégicos de futuro que deberán ser abordados por Gipuzkoa en colaboración con la sociedad civil y los principales agentes e instituciones territoriales . Desde el punto de vista metodológico, Etorkizuna Eraikiz responde a tres objetivos importantes:

1. Definición de los proyectos estratégicos concretos que es necesario abordar en los próximos diez años en el territorio Gipuzkoano.

2. Experimentar y aplicar con la colaboración de las empresas, la sociedad civil y las instituciones del territorio Gipuzkoano una serie de proyectos económicos, políticos, sociales y culturales que ayuden a orientar las necesidades y desafíos territoriales

3. Construir un nuevo modelo de gestión público más abierto, transparente $\mathrm{y}$ colaborativo.

Estas ideas básicas han derivado en el diseño y elaboración de una serie de iniciativas innovadoras asentadas sobre dos importantes pilares y una serie de medidas transversales:

Gipuzkoa Lab: centrado en el diseño de un Laboratorio para la implementación y evaluación de una serie de experiencias piloto para su posterior incorporación a las políticas públicas.

Gipuzkoa Taldean: orientando a la elaboración de un Plan Estratégico para Gipuzkoa 2026 desde la creación de un Think Tank centrado en la reflexión acerca de las necesidades económicas, políticas, sociales y culturales estratégicas para Gipuzkoa, desde una aproximación compartida y contrastada con agentes empresariales, de la administración pública y la sociedad civil.
Una serie de ejes transversales concretados en tres sub-proyectos: Nazioarteratze Sarea (Internacionalización), Ikergune (Investigación) y Gizarteatze Prozesua (Integración/Socialización).

\section{Gipuzkoa Lab}

Gipuzkoa Lab es el núcleo de Etorkizuna Eraikiz y está basado en la creación de un laboratorio de experiencias avanzadas de futuro para impulsar proyectos de interés prioritario en diferentes ámbitos estratégicos. Este laboratorio divide sus actividades entre la identificación de los mencionados proyectos para ser contrastados con los agentes económicos y sociales del territorio; la integración de estos proyectos en los programas y políticas públicas de la de la Diputación Foral; y finalmente, el diseño de las estrategias de intervención, las experiencias piloto y su aplicación.

Este Laboratorio es de una naturaleza fundamentalmente práctica y tiene por objetivo transformar las empresas, las instituciones públicas y el tercer sector de Gipuzkoa en Laboratorios donde poder experimentar, ensayar y aplicar nuevas políticas de carácter económico, social y cultural. Es este contexto, Gipuzkoa Lab funciona como un Laboratorio de Experimentación Activa en colaboración con la sociedad civil y los distintos agentes del territorio, con el propósito de incorporar la co-creación y codiseño de sus diferentes resultados a las políticas públicas del territorio gipuzkoano.

Los ámbitos temáticos abordados por Gipuzkoa Lab se dividen cuatro áreas estratégicas:

1. La participación de las personas en las empresas: La incentivación de este proceso de participación tiene múltiples efectos positivos: por un lado, fijar los centros de decisión al territorio gipuzkoano como vía para evitar su deslocalización; y por otro lado, hacer a las personas trabajadoras en la empresa participes de la gestión y la toma de decisiones estratégicas, mejorando así la competitividad de las empresas gipuzkoanas. En este contexto, es importante mencionar que Gipuzkoa es un territorio con una gran tradición participativa y un capital social fuerte, con presencia de cooperativas y sociedades laborales para lo cual es preciso reforzar los modelos existentes desde nuevas vías para la participación activa. 
2. Igualdad, conciliación y corresponsabilidad: Este eje aborda la importancia de la vida laboral y familiar, y la igualdad de oportunidades en la empresa, centrándose en la reducción de la brecha salarial entre hombres y mujeres, la escasa presencia de las mujeres en puestos de dirección y la precariedad del empleo femenino en el territorio. Todo ello desde la búsqueda experimental de fórmulas capaces de mejorar la conciliación de la vida familiar, personal y laboral, promoviendo así la corresponsabilidad.

3. Atención a mayores y grupos vulnerables: Esta área se divide en dos importantes proyectos: el primero se enmarca en el diseño de un Centro de referencia para la innovación y la mejora de la calidad de los recursos dirigidos a la atención de las personas mayores; el segundo, está basado en el diseño de cuatro experiencias piloto en cuatro comarcas del Territorio para promover la solidaridad vecinal y el voluntariado de proximidad en centros para el cuidado de personas mayores, proyectos de responsabilidad social con las empresas, y proyectos de voluntariado con los centros escolares.

4. Contenidos audiovisuales en Euskera: Esta línea de trabajo del laboratorio tiene como propósito la promoción de la producción y creación audiovisual en euskera. Observadas las dinámicas de consumo y las posibilidades de los limites de la creación en euskera, el objetivo de esta iniciativa radica en el diseño de experiencias piloto orientadas a la capacitación y transformación. De igual manera pretende poner en vías de desarrollo y transformación nuestras propias estructuras y actividades, para así cambiar las costumbres de consumo audiovisual en la ciudadanía, desde la perspectiva de país y el idioma (la lengua).

\section{Gipuzkoa Taldean}

Gipuzkoa Taldean, consiste en la formulación y desarrollo de un Plan Estratégico para Gipuzkoa en el 2026, desde la reflexión y puesta en marcha de una serie de proyectos. Estas preguntas se centran en la elaboración de un documento de base para saber en qué situación se encuentra el territorio, hacia dónde quiere cambiar, cómo es posible abordar el cambio que exige pasar de un estadio al siguiente y cuáles son los pasos que es necesario seguir para la transformación.

Para responder a estas preguntas, Gipuzkoa Taldean cuenta con la incorporación de una nueva generación de personas involucradas en los procesos de toma de decisiones de las empresas, organizaciones y asociaciones de Gipuzkoa que están en contacto diario, pero que, hasta el momento, se han encontrado alejadas de la realidad institucional del propio territorio. En este marco, Gipuzkoa Taldean constituye un Think Tank orientado a reflexionar sobre las necesidades económicas, políticas, sociales y culturales estratégicas para Gipuzkoa desde una aproximación compartida y contrastada.

Esta estructura establece cuatro áreas y grupos de trabajo en el año 2016 entre los cuales se incluyen:

- La promoción económica y turística del territorio para mejorar su competitividad y atractivo.

- La promoción de la educación, la cultura y la igualdad lingüística desde el conocimiento y la identidad territorial.

- La promoción del bienestar a través del diseño de nuevas políticas sociales.

- Y, finalmente, el apoyo a la movilidad, el medioambiente y las infraestructuras, desde el fomento de un territorio sostenible.

\section{Los ejes transversales}

Los ejes transversales a la estrategia descrita incluyen tres importantes iniciativas:

\section{Nazioarteratze Sarea (Internacionalización de la red)}

Es una estrategia de internacionalización a partir de la cual se pretende aprender de otros casos de éxito y experiencias de referencia desde la creación y acceso a nuevas redes y foros internacionales. Se trata de un sistema que busca aprender, comprender, analizar e incorporar otras iniciativas a nivel internacional desde dos importantes premisas:

- Analizar experiencias de éxito en países avanzados.

- Aplicar estas experiencias en Gipuzkoa.

- Integrar Etorkizuna Eraikiz en foros y redes internacionales. 
- Compartir y exportar las experiencias propias de Etorkizuna Eraikiz

\section{Ikergune (Investigación)}

Es una iniciativa que busca implicar al Sistema Universitario Gipuzkoano a través de sus cuatro universidades-Universidad del País Vasco (UPV/EHU), Universidad de Deusto, Mondragon Unibesitatea y Tecnun-, en el proceso de reflexión, debate y divulgación de las diversas acciones incluidas en el programa Etorkizuna Eraikiz. Este eje busca establecer una serie de programas de investigación centrados en cuatro objetivos importantes:

- Responder a preguntas útiles y relevantes para la sociedad Gipuzkoana.

- Realizar un proceso sistemático y continuo de evaluación y contraste de las prácticas políticas y los procesos organizacionales e institucionales con territorios líderes en estos campos.

- Divulgación de los resultados obtenidos.

- Participar en foros de debate para contrastar y co-crear nuevos programas de acción e investigación con distintos usuarios.

\section{Gizarteratze prozesua (Proceso de integración/socialización)}

acciones orientadas a la promoción de procesos de debate, difusión y socialización de los principales retos del territorio de Gipuzkoa, así como de las experiencias y resultados obtenidos, fomentando un proceso de aprendizaje y divulgación de conocimiento dentro de la sociedad gipuzkoana. Todo ello con el propósito de retroalimentar todo el proceso buscando:

- Compartir con los ciudadanos los valores y el proceso de trabajo a través de acciones colaborativas y co-diseño de nuevos proyectos.

- Implicar a los ciudadanos en nuevas dinámicas de participación política.

- Compartir con las empresas y las organizaciones Gipuzkoanas las experiencias, el desarrollo y los resultados obtenidos.

\section{Conclusiones}

El diseño del programa Etorkizuna Eraikiz constituye un pilar central en el tránsito hacia una nueva cultura institucional en la forma de gestionar y administrar el futuro de la gobernanza política del territorio Gipuzkoano. Si bien aún es pronto para valorar el impacto que este programa tendrá en los años venideros, su razón de ser se inscribe en un compromiso más ambicioso y complejo que, a través de estructuras metagubernamentales más estables, sea capaz de erigir en el medio-largo plazo un nuevo proceso de institucionalización basado en modelos y prácticas colaborativas más amplios. A pesar de las dificultades y la complejidad de estas estructuras, el arraigo y la necesaria implantación de una nueva cultura política es fundamental para el futuro institucional del territorio tanto desde el punto de vista discursivo como práctico por tres importantes razones:

En primer lugar, la representación conceptual del lenguaje que da sentido a las prácticas en la construcción del conocimiento experto sobre el espacio territorial; segundo, las prácticas y los planes de acción que se derivan de este lenguaje influenciando a los agentes e instituciones encargados de implementarlos; y, finalmente, las normas y los valores que guían las distintas acciones sociales, decidiendo cuáles son relevantes y cuáles son descartables y, por tanto, configurando dinámicas de poder hegemónicas que gobiernan los factores normativos $y$ conceptuales desde los que se construyen las políticas, aplicando cada discurso a su práctica espacial concreta (Richardson \& Jensen, 2004: 57$65)$.

De este modo, el análisis del diseño del programa Etorkizuna Eraikiz nos brinda la posibilidad de conocer los discursos y prácticas meta-gubernamentales que configuran la nueva construcción del futuro entramado institucional del territorio Gipuzkoano. En este contexto, cada estructura social suscita el pensamiento sobre las razones para su existencia, sus mecanismos de acción y comunicación, la configuración de sus entramados de poder, y el efecto de todos ellos a la hora de construir políticas y escenarios centrados en el fortalecimiento de la sociedad organizada y el aumento del proceso de legitimación política. Todo ello, a través de nuevos mecanismos de colaboración $\mathrm{y}$ 
representación política orientados a resolver los problemas de la sociedad Gipuzkoana, creando las herramientas necesarias para poder orientar y decidir sobre su futuro.

En este contexto, rescato aquí las nociones elementales de la teoría de la estructuración en las que la acción y la estructura se interpelan mutuamente, es decir, aquellas "reglas y recursos envueltos recursivamente en instituciones" que estructuran y a la vez son estructurados a través de las acciones y sistemas sociales de las sociedades a las que pertenecen, en un tiempo y un espacio dados. Acciones reproducidas por agentes e instituciones concretos que "aplican reglas y recursos en la diversidad de contextos de acción”, interactuando e influyéndose recíprocamente (Giddens, 2006: 60-61).

En este sentido, entre la denominada "conciencia discursiva y práctica no hay separación; existen sólo las diferencias entre lo que se puede decir y lo que en general simplemente se hace" (Giddens, 2006: 44). Dicho esto, conocer los motivos que subyacen a cada acción del discurso institucional Gipuzkoano es un objetivo prioritario; es por ello que el análisis del propio diseño del programa Etorkizuna Eraikiz va más allá de la implantación de una simple estrategia de acción política, por cuanto se inscribe en un proceso más amplio y ambicioso de reestructuración de la cultura política institucional de este territorio.
Por tanto, el objetivo último de una estrategia como la mencionada sería instituir un proceso mediante el cual, siguiendo a Loureau, "las fuerzas sociales instituyentes" terminen por "construir formas sociales codificadas e instituidas jurídicamente" (Loureau, 1979: 73). $\mathrm{Su}$ éxito radicará en la posibilidad de desarrollar acciones continuistas que bien a través de GipuzkoaTaldean o del futuro desarrollo del Plan Estratégico para el 2026 inscrito en esta última medida, sean capaces de conservar las perspectivas meta-gubernamentales propuestas en Etorkizuna Eraikiz.

Dicho esto y para concluir, siguiendo a Bill y Hardgrave (1992), la progresiva estructuración institucional del ámbito público ha debido de ir solventando distintos problemas: identidad, legitimidad, participación, penetración $\mathrm{y}$ distribución. De esta manera, "la vida púbica" deja de ser una mera Ágora pública donde debatir y acordar las cuestiones públicas para convertirse también en objeto de la economía política, un ámbito relacionado con los recursos y la actuación política. Una vez definidas estas metas a través de los procesos de planificación estratégica y programas como Etorkizuna Eraikiz, solamente queda institucionalizar los medios necesarios para crear las garantías que aseguren sus sostenibilidad en el futuro (Lechner, 1988).

\section{Referencias}

Agranoff, R., \& McGuire, M. (2003). Collaborative Public Management. New Strategies for Local Governments. Washington D.C.: Georgetown University Press.

Alexander, E. R. (1995). How Organizations Act Together: Interorganizational Co-ordination in Theory and Practice. Amsterdam: Overseas Publishers Association.

Atkisnon, M. M., \& Coleman, W. (1992). Policy Networks, policy communities, and the problems of Governance. Governance: An International Journal of Policy, Administration and Institutions, 5(2), 154-180.

Barlett, D., \& Dibben, P. (2010). Public Sector Innovation and Entrepreneurship: case studies from local government. Local Government Studies, 28(4), 107-121.

Bason, C. (2010). Leading Public Sector Innovation. Co-creting for a Better Society. Bristol: The Policy Press.

Beuir, M. (2012). Governance: A very short introduction. Oxford: Oxford University Press.

Borras, S., \& Jacobsson, K. (2004). The open method of coordination and new governance patterns in the EU. Journal of European Public Policy, 11(2), 185-208.

Bouckaert, G. (2012a). Trust and Public Administration. Administration, 60(1), 91-115.

-. (2012b). Reforming the Performance and Trust: Some Reflections. The NISPAcee Journal of Public Administration and Policy, 5(1), 9-20.

Bovaird, T., \& Löffler, E. (2009). Public Management and Governance. London: Routdlege.

Bukowski, J., Piattoni, S., \& Smyrl, M. (Eds.). (2003). Between Europeanization and Local Societies: the space for territorial governance. Oxford, UK: Rowmen \& Littlefield. 
Camagni, R. (2002). On the concept of Territorial Competitiveness: Social or Misleading? Urban Studies, 39(13), 2395-2411.

Candy, P., C. (1991). Self-direction for Lifelong Learning: A comprehensive guide to theory and practice. San Francisco: ERIC.

Castells, M. (1997). La Era de la Información. Vol 1. La Sociedad Red. Madrid: Alianza Editorial.

Christopoulos, S., Horvath, B., \& Kull, M. (2012). Advancing the Governance of Cross-Sectoral Policies for Sustainable Development: A Metagovernace Perspective. Public Admistration and Development, 32(3), 305323.

Crevoiser, O. (2004). The Innovative Milieus Approach: Toward a Territorialized understanding of the Economy? Economic Geography, 80(4), 367-379.

Crouch, C. (2011). The Strange Non-death of Neo-Liberalism. Cambridge: Polity.

Crozier, M., Huntington, S. P., \& Watamuki, J. (1975). The Crisis of Democracy. Report on the Governability of Democracies to the Trilateral Commission. New York: New York University Press.

Dickinson, R. E. (1964). City and Region: a geographical interpretation. London: Routdlege \& Keagan Paul.

Diputación Foral de Gipuzkoa, F. d. (2016). Plan Estratégico de Gestión 2015-2019. Donostia-San Sebastián: Diputación Foral de Gipuzkoa.

Duit, A., \& Galaz, V. (2008). Governance and Complexity: Emerging Issues for Governance Theory. Governance: An International Journal of Policy, Administration and Institutions, 21(3), 311-335.

Emerson, K., Nabatchi, T., \& Balogh, S. (2011). An Integrative Framework for Collaborative Governance. Journal of Public Administration, Research and Theory, 22(1), 1-29.

Engberg, L. A., \& Larsen, J. N. (2010). Context-oriented Meta-Governance in Danish Urban Regeneration. Planning Theory and Practice, 11(4), 549-571.

Estensoro, M., \& Zurbano, M. (2010). La Innovación Social en la Gobernanza Territorial: Los casos de Innobasque y de las Redes Comarcales en el País Vasco. Ekonomiaz 74(160-177).

European, Commission. (2001). European governance: a white paper. Luxembourg.

Faludi, A. (2012). Multi-level (territorial) governance: Three criticisms. Planning Theory and Practice, 13(2), 197211.

Ferlie, E., Ashburner, L., Fitzerald, L., \& Pettigrew, A. (Eds.). (1996). The New Public Management in Action. New York: Oxford University Press.

Field, J. (2000). Lifelong learning and The New Educational Order. London: ERIC.

Foxell, S. (2015). Design as a Policy Tool. Building Research and Information, 43(4), 553-555.

Fukuyama, F. (2013). What is Governance? Governance: An International Journal of Policy, Administration and Institutions, 26(3), 347-368.

Giddens, A. (2006). La Constitución de la Sociedad: Bases para la teoría de la estructuración. Buenos Aires: Amorrortu Editores.

Gobierno Vasco. (2014). Informe sobre el análisis y funcionamiento institucional en la Comunidad Autónma de Euskadi. Vitoria-Gasteiz: Gobierno Vasco.

Harvey, D. (2007). Breve historia del neoliberalismo. Barcelona: Akal.

Jessop, B. (1998). The Rise of Governance and the Risk of Failure: The Case of Economic Development. International Social Science Journal, 50(155), 29-45.

-. (2002). Governance and Metagovernace: On reflexivity, Requisite Variety and Requisite Irony from http://complanes.au.uk/sociology/papers/Jessop-Governance-and-Metagorvernace.pdf

-. (2003). Governance and Metagovernance: On Reflexivity, Requisite, Variety and Irony. In H. P. Bang (Ed.), Governance as Social and Political Communication (pp. 142-172). Manchester: Manchester University Press.

-. (2004). Multilevel Governance and Multilevel Metagovernance. In I. Bach \& M. Flinders (Eds.), Multi-level Governance (pp. 49-74). Oxford: Oxford University Press.

Jones, C., Hesterly, W. S., \& Bourgatti, S. P. (1997). A General Theory of Network Governance: Exchange Conditions and Social Mechanisms. The Academy of Management Review, 22(4), 911-945.

Keating, M. (1997). The Invention of Regions: political re-estructuring and territorial government in Western Europe. Environment and Planning C: Government and Policy, 15(4), 383-398.

Keating, M. (1998). The New Regionalism in Western Europe. Northhampton: Edward Elgar Publishing.

Keating, M. (2002). Governing Ctities and Regions: Territorial Restructuring in a Global Age. In A. J. Scott (Ed.), Global City Regions. Trends, Theory and Policy. New York: Oxford University Press.

Kjaer, A. M. (2004). Governance. Cambridge U.K.: Polity.

Lathrop, D., \& Ruma, L. (Eds.). (2010). Open Government. Sebastopol: O'reilly. 
Lechner, N. (1988). Los Patios Interiores de la Democracia: Subjetividad y Política. FLACSO: Santiago de Chile.

Lidström, A. (2007). Territorial Governance in Transition. Regional and Federal Studies, 17(4), 499-508.

Lipset, S. M. (1959). Some Social Requisites of Democracy. Economic Development and Polictical Legitimacy. The American Political Science Review, 53(1).

Loureau, R. (1979). El Estado y el Inconsciente. Barcelona: Editorial Kairos.

Luhmann, N. (1982). The World Society as a Global System. International Journal of General Systems, 8(3), 131138.

-. (1995). Social Systems. Stanford: Stanford University Press.

Luna, A. (2015). La Innovación de la Metrópoli: de Bilbao a Oresund y San Diego. Leioa: UPV/EHU.

Martin, R., Kitson, M., \& Tyler, P. (Eds.). (2008). Regional Competitiveness. London \& NY: Routdlege.

McLaughlin, K., Osborne, S. P., \& Ferlie, E. (Eds.). (2002). New Public Management. Current trends and future prospects. . ondon \& NY: Routdlege.

Meuleman, L. (2008). Public Managmenet and Metagovernace of Hierarchies, Networks and Markets. PhysicaVerlag: Heidelberg.

Moulaert, F. (2000). Globalization and Integrated Area Development in European Cities. New York: Oxford University Press.

Moulaert, F., \& Sekia, F. (2003). Territorial Innovation Models: a critical survey. Regional Studies, 37(3), $289-302$.

Moynihan, D. P. (2009). The Network Governance of Crises Response: Case Studies of Incident Command Systems. Journal of Public Administration, Research and Theory, 19(4), 895-915.

Mulgan, G., \& Albury, D. (2003). Innovation in the Public Sector. Retrieved from London.

O'Flynn, J., \& Wanna, J. (2008). Collaborative Governance: a new era of public policy in Australia? Canberra: ANU Press.

Osborne, S. P. (2008). The New Public Governance. Public Management Review, 8(3), 377-387.

Osborne, S. P. (Ed.). (2010). The New Public Governance? Emerging theories on the theory and practice of Public Governance. New York: Routdlege.

Papadopoulos, Y. (2007). Problems of Democratic Accountability in Network and Multilevel Governance. European Law Journal, 13(4), 469-486.

Penin, L., Staszowski, E., \& Brown, S. (2015). Teaching the Next Generation of Transdisciplinary Thinkers and Practionners of Design-based Public and Social Innovation. Design and Culture. The Journal of Design Studies Forum, 7(3), 441-450.

Posner, R. A. (2010). The Crisis of Capitalist Democracy. Cambridge, M.A. : Harvard University Press.

Provan, K. G., \& Kervis, P. (2008). Modes of Network Governance: Structure Managment and Effectiveness. Journal of Public Administration, Research and Theory, 18(2), 229-252.

Rhodes, R. A. W. (1996). The New Governance: Governing without Government. Political Studies, 44(4), 652-667.

-. (1997). Understanding Governance: policy networks, governance, reflexivity, and accountability. Maidehad, G.B.: Open University Press.

-. (2007). Understanding Governance: Ten Years On. Organization Studies, 28(08), 1243-1264.

Richardson, T., \& Jensen, O. B. (2003). Linking discourse and space: Towards a cultural sociology of space in analysing spatial policy discourses. Urban Studies, 40(1), 7-22.

Robertson, R. (1995). Globalisation or Glocalization? The Journal of International Communication, 1(1), 33-52.

Sorensen, E. (2006). Metagovernance: The Changing Role of Politicians in the Process of Democratic Governance. American Review of Public Administration, 36(1), 98-114.

Sorensen, E., \& Torfing, J. (2007). Theories of Network Governance. New York: Palgrave MacMillian.

-. (2012). Introduction: Collaborative Innovation in the Public Sector. The Innovation Journal: The Public Sector Innovation Journal, 17(1).

Stoker, G. (1995). Regime Theory and Urban Politics. In David Judge, G. Stoker \& H. Wolman (Eds.), Theories of Urban Politics (pp. 54-72). London: Sage Pubications.

Torfing, J. (2016). Collaborative Innovation in the Public Sector. Washington DC: Georgetown University Press.

Torfing, J., Peters, G., Pierre, J., \& Sorensen, E. (2012). Interactive Governance. Advancing the Paradigm. New York: Oxford University Press.

Valdaliso, J. M., \& Wilson, J. R. (Eds.). (2015). Strategies for Shaping Territorial Competitiveness. NY: Routdlege.

Windrum, P., \& Koch, P. (Eds.). (2008). Innovation in Public Sector Services: Entrepreneurship, Creativity, and Management. Northampton, MA: Edward Elgar.

World Bank. (2000). Performing public institutions and strenghtening Governance. A world bank strategy. Washington D.C. 